\title{
PHƯƠNG PHÁP GIẢNG DẠY LÝ THUYÊT KẾT HỢP MÔ PHỎNG - THỰC HÀNH CỦA SINH VIÊN NGÀNH ĐIỆN TỦ
}

\author{
Võ Thanh Tùng(*), Võ Văn Ân ${ }^{(* *)}$ \\ (*) Thạc sĩ. Truoòng Đại học Công nghệ Đồng Nai. Email: vothanhtung@dntu.edu.vn. \\ (**)Thạc sĩ. Truờng Đại học Kinh tế Kỹ thuật Bình Duoong.
}

DOI: $\underline{10.37550 / \mathrm{tdmu} . \mathrm{CFR} / 2021.01 .119}$

\section{Tóm tắt}

Nghiên cưu việc sử dụng các phưong pháp hoc tập với sụ hồ trợ của máy tính để tăng cuờng tính tích cực học tập và tạo hứng thú để sinh viên ngành Điện tử học tập tốt hơn các môn điện tư tuơng tụ và kỹ thuật số. Khi các môn Điện tử tuong tụ và Kỹ thuật số được thiết kế lại trong tùng khóa học, sinh viên sẽ có hứng thú hơn trong việc học tập. Ngoài ra sinh viên còn có thêm động lực thực hiện các bài tập và chuẩn bị cho các bài học mới đurợc dựa trên phương pháp mà Giảng viên cung cấp nhu các công cu phần mềm mô phỏng (1) nhằm tăng tính tương tác. Tốt hơn nũa, một số bài tập về mạch điện-điện tử sẽ được giao cho sinh viên thực hiện duới dạng mô phỏng trước và sau đó mói thục hiện trên các linh kiện thực tế. Hay nói cu thể hơn là bài tập dược thiết kế -xây dựng tù lý thuyết trên bài giảng, tiếp đến là mô phỏng trên máy tính và cuối cùng sẽ thực hiện mạch trên mô hình thưc tế. Trên đây là phwơng pháp giảng dạy được nghiên cứu để úng dụng CDIO vào học tập cho sinh viên ngành Điện tư tại trương Đại học Công Nghệ Đồng Nai. Trong quá trình hoc tập, sinh viên có thể tụ do sáng tạo, làm ra được nhũng sản phẩm học tập của riêng mình và tự đánh giá được quá trình học tập và tiếp thu của bản thân.

Từ khóa: lĩnh vục giảng dạy điện tử, mô phỏng mạch điện tử, phuoong pháp học điện tủ

\section{1. Đặt vấn đề}

Tiềm năng của phương pháp giảng dạy "e-learning" (được hiểu là học tập và hướng dẫn dựa trên web, mô phỏng tương tác cũng như làm các bài kiểm tra trực tuyến) là nâng cao khả năng học tập tích cực trong giáo dục chuyên ngành kỹ thuật cho đến nay không phải là vấn đề chính trong CDIO. Mặc dù tính tích cực của các hoạt động dạy và học dựa trên kết quả đánh giá học tập là một phần của tiêu chuẩn $\mathrm{CDIO}$ (tiêu chuẩn 8: học tập tích cực), nhưng mức độ phù hợp của phương pháp giảng dạy e-learning (7) đối với việc hỗ trợ các mục tiêu giáo dục này không được coi là một phần thuộc hệ thống trong khuôn khổ CDIO.

Lý giải rõ hơn về điều này vì phương pháp giảng dạy e-learning có thể bị che khuất bởi sự tập trung của CDIO về tầm quan trọng của trải nghiệm thực hành và thiết kế xây dựng 
chuẩn đầu ra. Tuy nhiên, tầm quan trọng của phương pháp giảng dạy e-learning thông qua các công cụ phần mềm mô phỏng cho thấy việc sử dụng các kỹ thuật mô phỏng có hỗ trợ của máy tính để nâng cao học tập tích cực và thúc đẩy sinh viên chuẩn bị cho bài học và bài tập ngay từ đầu. Trong một số ngành kỹ thuật hiện đại như công nghệ kỹ thuật điện tử và công nghệ thông tin, lĩnh vực mô phỏng và thử nghiệm trên máy tính đã khai thác rất nhiều vào các đề tài nghiên cứu, giảng dạy, và đây là một lý do chính để giới thiệu các kỹ thuật e-learning như mô phỏng và tương tác trực tuyến, giúp sinh viên có thể xây dựng mô phỏng hoạt động các mạch điện tử. Điều này không đồng nghĩa là khi học về điện, điện tử sinh viên sẽ không còn thi công, trải nghiệm các mạch điện, điện tử với các linh kiện thực tế.

\section{Các môn học liên quan chuyên ngành điện tử}

Việc đưa một số phương pháp e-learning vào các môn học nhập môn Điện tử và các môn cơ sở ngành, chuyên ngành là một trong nhiều phương pháp trong kế hoạch giảng dạy học tập áp dụng CDIO. nghiên cứu về e-learning(6) ứng dụng trong chuyên ngành điện tử, là một phần trong nỗ lực của khoa Công Nghệ nói riêng và toàn bộ DNTU trong việc áp dụng CDIO làm cơ sở cho tất cả các chuyên ngành đào tạo của nhà trường.

Hình 1 dưới đây cho thấy sự thay đổi về tổ chức lại cách thức giảng dạy các môn học trong ngành điện tử hiện tại (trái) và thực hiện cải tiến (phải). Các sự thay đổi cách thức giảng dạy áp dụng CDIO có liên quan đến các môn học về lập trình và kỹ thuật phần mềm không được đề cập trong bài báo cáo này.

Phương pháp cũ

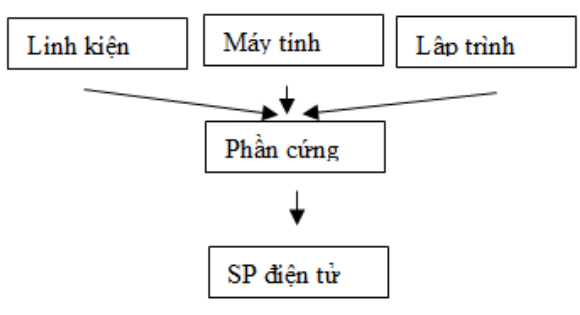

Phương pháp mới

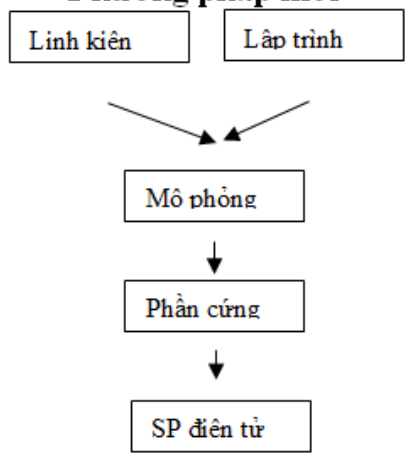

Hình 1. Một phần được tổ chức lại của kế hoạch học tập cho chuyên ngành điện tử

Trong kế hoạch học tập mới, các môn học kỹ thuật số, ngôn ngữ lập trình phần mềm $\mathrm{C}, \mathrm{C}++$ được dạy vào học kỳ thứ hai, có nghĩa là bây giờ sinh viên có thể tiếp cận chuyên ngành ngay từ môn học nhập môn chuyên ngành điện tử. Sinh viên đã biết về linh kiện điện tử và học một ngôn ngữ lập trình, VHDL có thể được giới thiệu như một ngôn ngữ khác nhằm mục tiêu thiết kế phần cứng. Đồng thời, mục đích của chúng tôi là giới thiệu một số vấn đề rất đơn giản giống như $\mathrm{CDIO}$, tức là các vấn đề phản ánh vòng đời của quá trình hình thành-thiết kế-triển khai-vận hành, trong các môn học Điện tử nơi sinh viên sẽ:

1) Thiết kế mạch sử dụng giấy và bút chì (tức là giải quyết vấn đề truyền thống).

2) Sau đó là "nguyên mẫu" mạch điện sử dụng các công cụ vẽ và mô phỏng sơ đồ. 
3) Xây dựng mạch điện tử thực tế bằng cách sử dụng bảng mạch, các thành phần và linh kiện điện tử.

\section{Phương pháp dạy học sử dụng 5 spice}

5Spice (14) là phiên bản cơ bản của phần mềm điện tử chuyên nghiệp PSpice, mặc dù phiên bản cơ bản vẫn chưa được sử dụng rộng rãi như phiên bản chuyên nghiệp. Phiên bản này đã được sử dụng để tạo ra các sơ đồ như một bước đầu tiên hướng tới các cấu trúc giống CAD nâng cao hơn cho các mạch vi điện tử VLSI(11). 5Spice không phải là mã nguồn mở, nhưng nó cho phép sử dụng miễn phí cho các mục đích giáo dục trong việc mô phỏng các mạch điện tương tự. Trong ví dụ (hình 2) một mạch RC thụ động đã được xây dựng để minh họa cách điện áp hình thành trong quá trình nạp tụ điện $(\mathrm{C} 1)$.

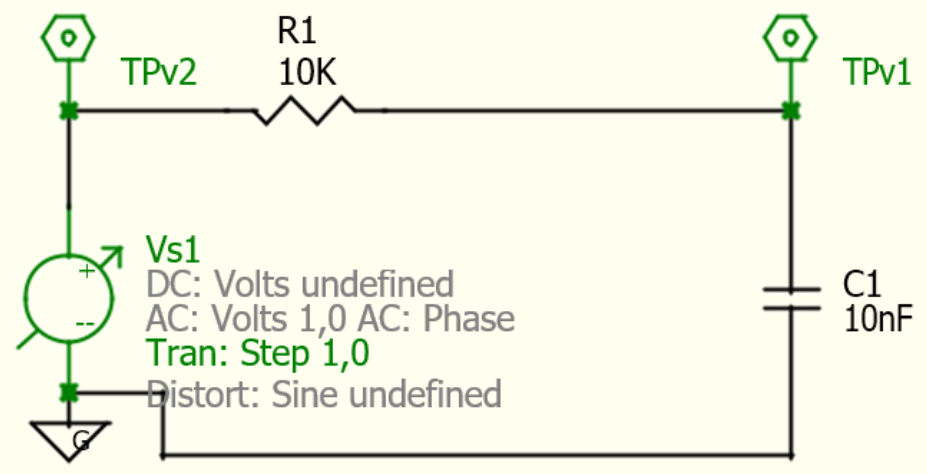

Hinh 2. Một mạch RC đơn giản được xây dựng trong 5Spice.

Hoạt động của tụ điện có thể được nghiên cứu thông qua phân tích quá độ của mạch như được hiển thị trong đồ thị của điện áp (đo bằng TPv1) dưới dạng hàm thời gian. Sau một thời gian mức điện áp đạt hiệu điện thế trên nguồn (đo bằng TPv2). Đồ thị (hình 3) thể hiện tầm quan trọng của yếu tố thời gian trong điện tử, vì phải mất vài lần hằng số thời gian cụ thể của mạch $(\tau=\mathrm{RC})$, để tụ điện được sạc đầy. Sự chậm trễ này về nguyên tắc là nguyên nhân gây ra hạn chế đối với tốc độ có thể của chip máy tính, tức là tần số xung nhịp. 


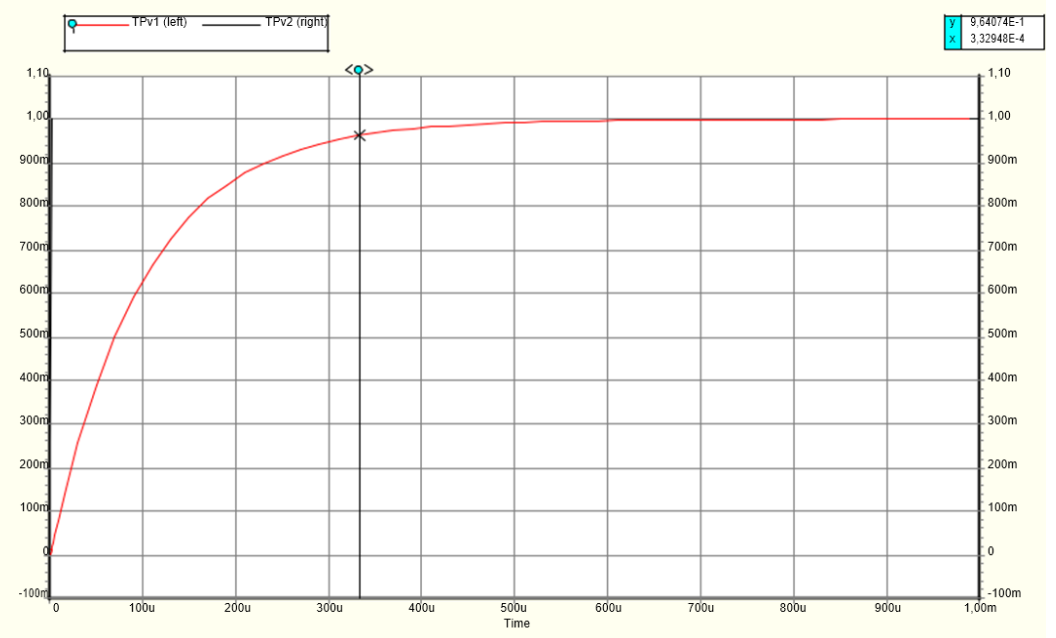

Hinh 3. Đồ thị của điện áp dưới dạng hàm thời gian trên tụ điện.

Vì vậy, ví dụ này được chọn do tính phức tạp, trong vấn đề trễ tương tự đặc trưng cho mô hình chuyển mạch RC (5) của các mạch CMOS được sử dụng trong các mạch kỹ thuật số và bộ vi xử lý. Các mạch này được giới thiệu trong khóa học điện tử mới và ví dụ (tức là vấn đề về thời gian trễ) có thể được sử dụng để liên kết với hai môn học chính của ngành điện tử là điện tử tương tự và điện tử kỹ thuật số. Hình 4 cho thấy một slide PowerPoint từ phần giới thiệu về chuyển mạch trong kỹ thuật số minh họa vấn đề cơ bản này.

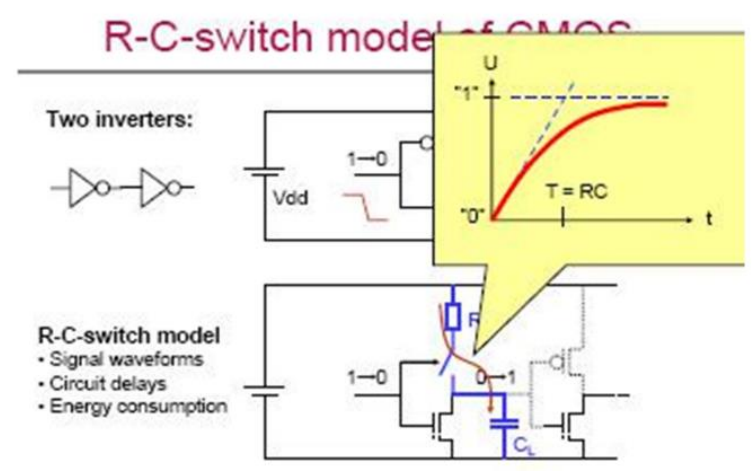

Hinh 4. Slide PowerPoint tì̀ phần giới thiệu về chuyển mạch trong kỹ thuật số.

Bằng cách sử dụng 5Spice, sinh viên có cơ hội xây dựng và mô phỏng hoạt động của một mạch để sau này sinh viên sẽ thực hiện thi công trong phòng thí nghiệm. Hướng dẫn cho một bài tập trong 5 Spice có thể là:

"Xây dụng một mạch ảo trong 5Spcie có thể được sủ dụng để mô phỏng quá trình nạp xả của tu điện thông qua điện trở. Đo hiệu điện thế trên tụ điện duới dạng hàm thời gian. Cho điện trở $R=10 \mathrm{k} \Omega$ và điện dung $C=10 \mu \mathrm{F}$, và xem xét thông qua phân tích quá độ tại thời điểm đó điện áp trên tụ điện đạt mức ứng với 95\% giá trị cuối cùng của nó."

Trong biểu đồ do 5 Spice tạo ra (hình 3), thời gian được yêu cầu có thể được đọc trên biểu đồ. Ô nhỏ ở góc trên bên phải hiển thị tọa độ của giao điểm. Trong phần tiếp tục của bài tập nhỏ này, sinh viên cần được yêu cầu tính toán thời gian nạp xả và phân tích và sau đó họ sẽ có cơ hội xây dựng mạch điện tử trong phòng thí nghiệm và kiểm tra tín hiệu vật lý bằng máy hiện 
sóng.Các mô phỏng đơn giản kiểu này cũng có thể được sử dụng trong bối cảnh làm việc ở nhà với các bài tập tính toán (không cần thi công mạch và đo kiểm) để nâng cao hiểu biết về lý thuyết như được mô tả trong giáo trình môn học về các định luật về điện (ví dụ định luật Ôm) và mạch điện (ví dụ định luật Kirchhoff). Hy vọng rằng cách này sinh viên sẽ được cải thiện hơn trong phương pháp học tập cũng như thú vị hơn thông qua loại bài tập về nhà này. Thêm một cách khác là sinh viên làm việc nhóm trong khuôn viên lớp học, trường học kiểm tra hoạt động của các mạch ảo và đặc tính của các thành phần của chúng (chẳng hạn như tụ điện trong ví dụ của chúng tôi). Sinh viên sẽ dần dần xây dựng sơ đồ mạch điện và hiểu hoạt động của các linh kiện trong các loại mạch điện khác nhau, khi sinh viên tiếp xúc với nhiều ví dụ và buộc phải tìm hiểu vai trò và chức năng của các linh kiện điện tử khác nhau trong mạch điện. Các mạch ảo và chương trình phần mềm như 5 Spice rất phù hợp cho mục đích này, do đó có thể được sử dụng như một loại “mô phỏng gần thực tế” được hỗ trợ bằng máy tính.

5Spice cũng có thể được sử dụng cho các bài tập để đào tạo sinh viên trong việc lựa chọn các thành phần liên quan và ước tính kích thước phù hợp (điện trở, điện dung, dòng điện, v.v.). Trong phòng thí nghiệm, sinh viên đôi khi sẽ thi công sai các linh kiện và các mạch sẽ không hoạt động vì lựa chọn các thành phần và kích thước thuộc tính không đúng. Điều này cũng tạo thành một kinh nghiệm học tập. Các bài tập ảo cũng như thực hành trong phòng thí nghiệm là một cách để bù đắp cho sự thiếu kinh nghiệm của các sinh viên ngành điện tử.

\section{Các lợi ích khi sử dụng phương pháp giảng dạy e-learning}

Để chuẩn bị cho việc giới thiệu linh kiện điện tử cho sinh viên trong môn nhập môn chuyên ngành điện tử, chúng tôi đã nghiên cứu một số công cụ phần mềm tương ứng. Một tùy chọn là sử dụng phần mềm mã nguồn mở dựa trên java để đưa ra các hướng dẫn và câu hỏi trên web nhằm hỗ trợ bài tập về nhà của sinh viên, đồng thời làm công cụ trực quan cho việc giảng dạy trên lớp. Trong một số trường hợp, các chương trình dựa trên java có thể được nhúng vào các nền tảng e-learning như Moodle (15), canvas. Các nền tảng học tập trực tuyến này cũng sẽ hỗ trợ việc tạo ra các phòng học online và các phương tiện tương tác khác mà sinh viên có thể sử dụng để học tập trong khuôn viên trường hoặc tại nhà. Một lựa chọn khác nữa là hướng dẫn sinh viên tải xuống các chương trình nhỏ có thể được sử dụng miễn phí cho mục đích giáo dục (5Spice đã đề cập ở trên) để xây dựng và mô phỏng các mạch điện tử. Những phương tiện này nhằm thúc đẩy sinh viên tham gia vào học tập ở nhà và tăng cường học tập tích cực mọi lúc mọi nơi

Tuy nhiên, để xem xét tính hữu ích tiềm năng của e-learning, cần phải hiểu và phân tích "lợi ích" của e-learning như:

- Vai trò của hình ảnh hóa (tĩnh) so với hình ảnh động

- Vai trò của đồ họa 2-D so với đồ họa 3D

- Vai trò của tương tác (do người dùng kiểm soát) so với mô phỏng (dựa trên mô hình)

- Vai trò của đa phương tiện (ví dụ: phương tiện nghe nhìn) so với nhiều hình thức biểu diễn khác

\section{Sử dụng các applet java đơn giản so với các trình mô phỏng mạch nâng cao}


Với các lợi ích của e-learning như trên, giờ đây chúng ta có thể đánh giá tốt hơn các công cụ phần mềm khác nhau có thể được sử dụng để dạy và học trong chuyên ngành điện tử. Một ví dụ mạch điện được hiển thị bên dưới bằng cách sử dụng applet java Circuit Simulator (16).

Ví dụ về mạch RC có thể được sử dụng tương tác để hình dung quá trình nạp xả của tụ điện cũng như sự phóng điện của nó (bằng cách nhấp vào công tắc). Bên dưới mạch hoạt động trực quan bằng hình ảnh động (lưu ý các "electron" đại diện trên mạch) biểu đồ cho dòng điện (đường màu vàng) và điện áp (đường màu xanh lá cây) cho quá trình sạc và phóng điện tiếp theo của tụ điện. Nhiều mạch cấu hình sẵn khác của điện tử tương tự và kỹ thuật số có thể được chọn từ menu applet. Giá trị chương trình này là sự lặp lại của các loại mạch khác nhau đã được đề cập trong các bài giảng và giáo trình. Tuy nhiên, khả năng sinh viên tự xây dựng mạch của riêng mình là rất hạn chế trong loại applet này. Sự tương tác được giới hạn trong việc lựa chọn loại mạch và một vài cài đặt tham số bên ngoài (chẳng hạn như tốc độ mô phỏng và lựa chọn hiển thị đồ thị).

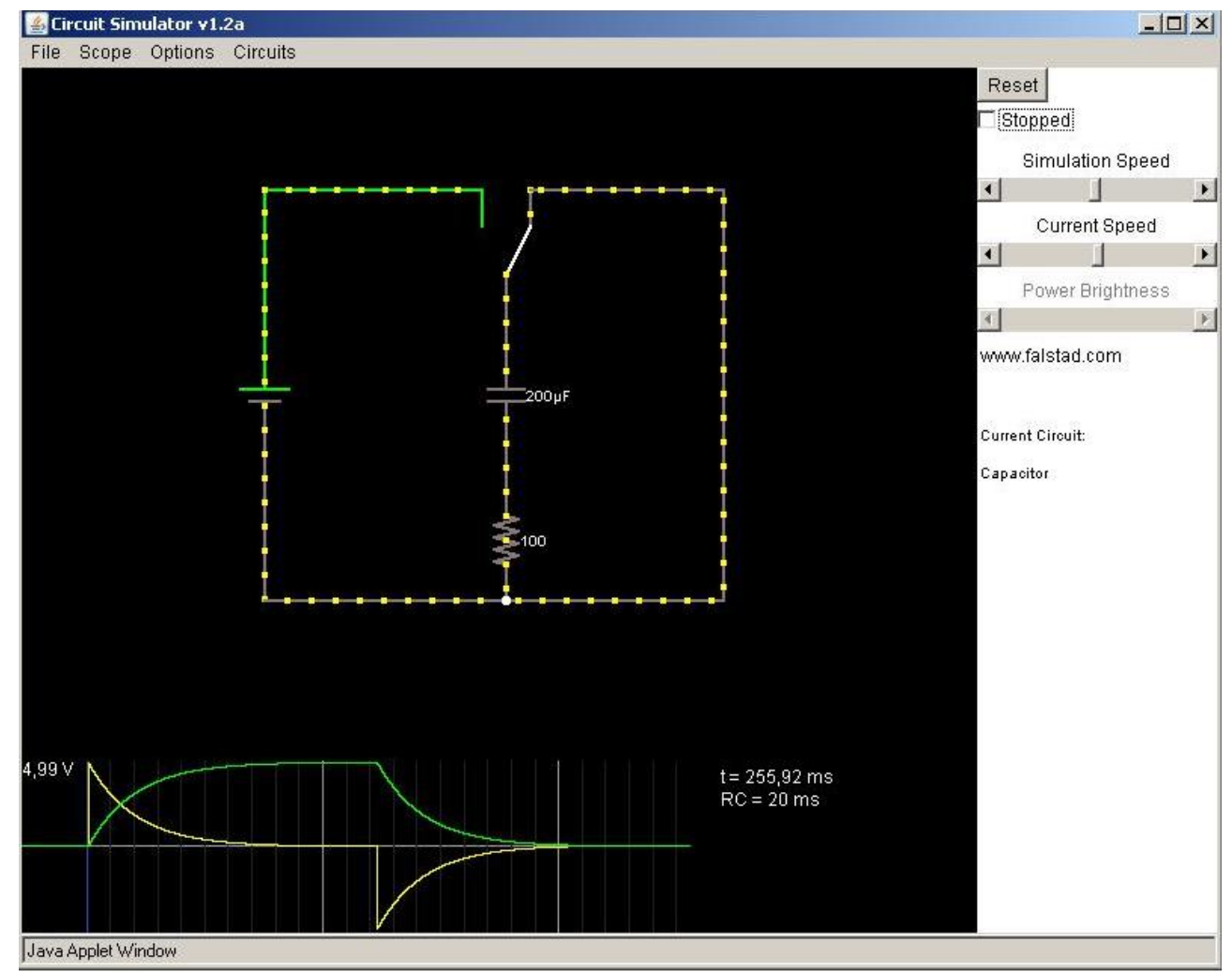

Hinh 5. Java applet mô phỏng một mạch RC đơn giản.

Điều này có nghĩa là một công cụ như thế này có thể được sử dụng làm hỗ trợ các mạch điện cơ bản để kiểm tra các thông số như dòng điện và điện áp trong các mạch xác định trước (như đã thảo luận ở trên), nhưng nó không hỗ trợ cho các thí nghiệm về một số mạch điện tử phức tạp. Với các môn học đòi hỏi mô phỏng các mạch điện phức tạp cần tính toán đo kiểm nhiều thông số sinh viên có thể sử dụng các công cụ nâng cao hơn như Logisim, Circuit Sandbox (18) cũng như 5Spice đã đề cập ở trên. Dưới đây là ví dụ về bộ cộng-trừ 4 bit được sử dụng để điều khiển màn hình led 7 đoạn. 
Trong các công cụ tiên tiến hơn, vẫn có thể thử các mạch điện tử được cấu hình sẵn, nhưng sinh viên cũng có thể xây dựng các mạch từ các linh kiện riêng lẻ và sau đó chạy chúng dưới dạng mô phỏng. Một số chương trình mã nguồn mở này dựa trên java và có thể được triển khai dưới dạng mô phỏng dựa trên web trong khi các chương trình khác yêu cầu sinh viên tải phần mềm xuống máy tính và cài đặt. Ví dụ về bộ cộng - trừ sẽ là một ví dụ điển hình về bài tập về môn kỹ thuật số, nơi học sinh tự xây dựng mạch và bảng giá trị tương ứng của nó.

Đối với các khía cạnh khác của e-learning đã đề cập ở trên, chúng tôi sẽ chỉ nhận xét về vấn đề đồ họa $2 \mathrm{D}$ và $3 \mathrm{D}$. Có ý kiến cho rằng vì có nhiều biến đồng thời (điện áp, dòng điện, công suất, v.v.) thường được hiển thị dưới dạng nhiều biểu đồ bên ngoài biểu diễn sơ đồ của mạch, một cải tiến khả thi về trực quan có thể là tích hợp các biểu đồ và sơ đồ để có được một biểu đồ 3D Biểu đồ sau đó được làm động để hiển thị hoạt động phụ thuộc vào thời gian của các mạch. Nguyên tắc của hoạt động là sơ đồ mạch đã cho được sử dụng làm sơ đồ $2 \mathrm{D}$ của một biểu đồ biểu thị cả điện áp và dòng điện. Sau đó, biểu đồ được "nâng" lên chiều thứ ba bằng cách mã hóa các mức điện áp của nút là chiều cao của nút và bằng cách mã hóa dòng điện qua đường dẫn như chiều cao của vùng bóng mờ bên dưới đường dẫn. Công suất (tích của hiệu điện thế và cường độ dòng điện) được biểu diễn dưới dạng mũi tên phía trên đồ thị mạch (hình 7). Hình ảnh hóa có thể được mô tả như một nỗ lực để hợp nhất cấu trúc liên kết mạch và các trạng thái biến đổi mạch thành một đồ thị duy nhất. Nó đã được triển khai dưới dạng một gói bổ trợ cho Mathematica (19) và do đó không được cung cấp miễn phí.

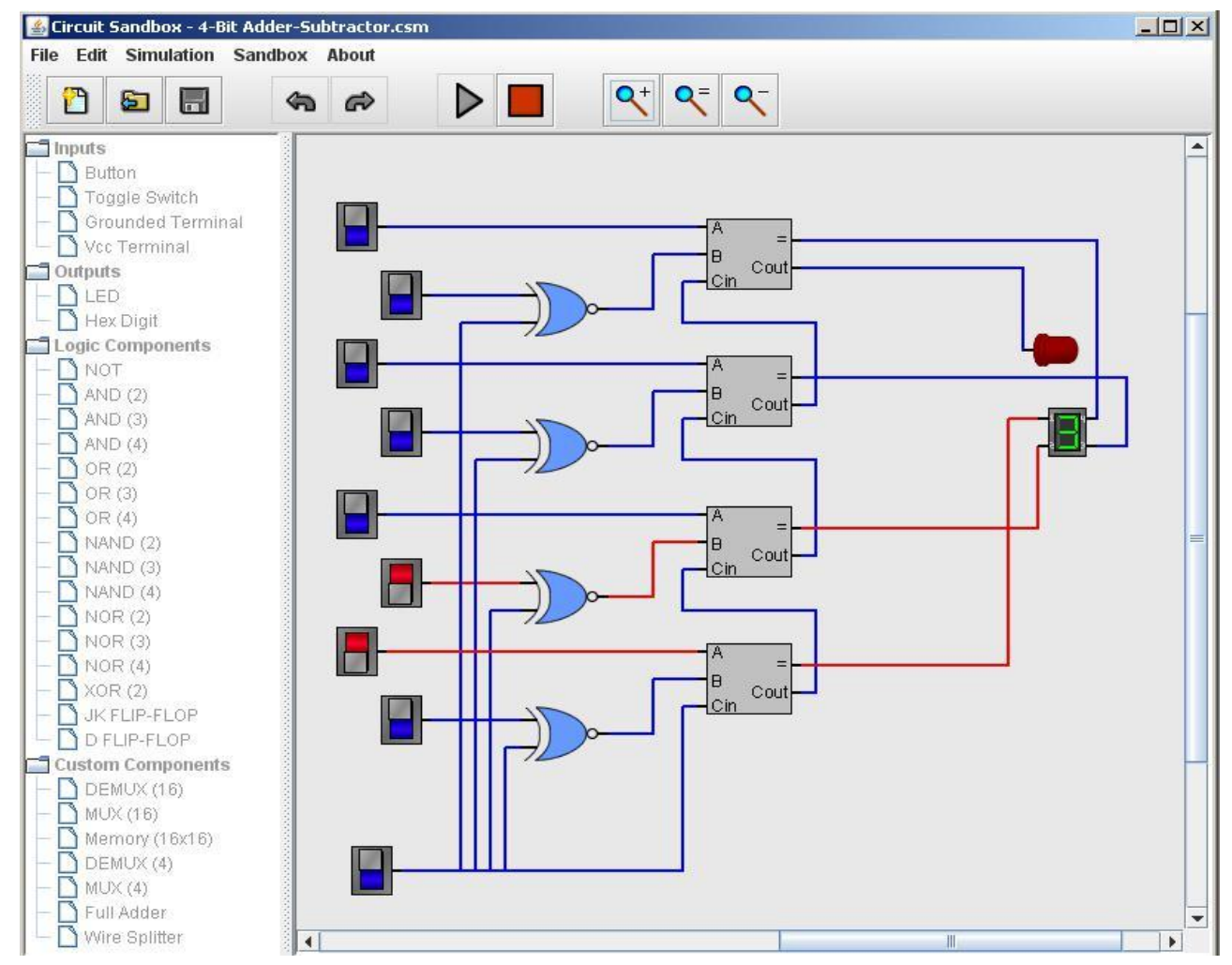

Hinh 6. Bộ cộng-trù 4 bit được sử dụng để điều khiển màn hình led 7 đoạn 


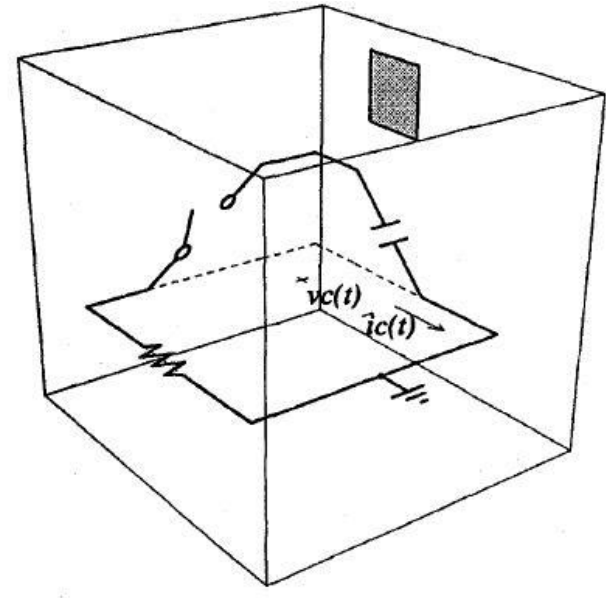

(a)

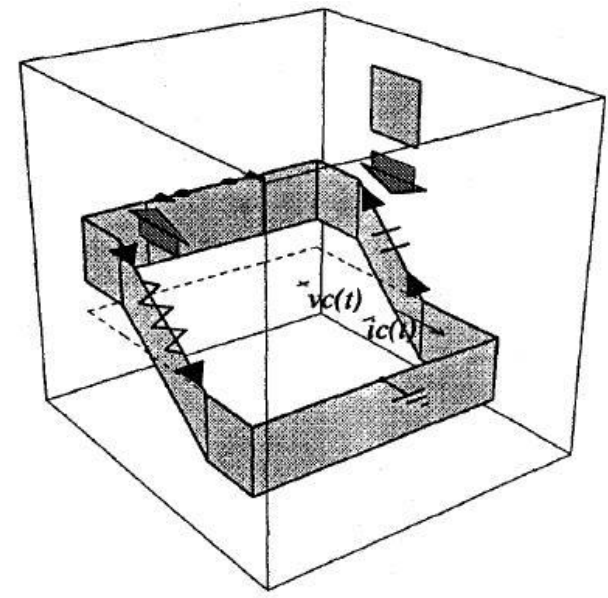

(b)

Hình 7. Hình ảnh $3 D$ về điện áp, dòng điện và công suất của một mạch $R C$ (a) trước khi đóng công tắc với năng lương ban đầu được lưu trũ trong tụ điện và (b) hình dung mạch ngay sau khi đóng công tắc điện.

Cuối cùng, chúng ta nên biết rằng áp dụng $\mathrm{CDIO}$ với việc học tập tích cực, nghĩa là sinh viên cần được tiếp xúc với những kinh nghiệm thực tế liên quan đến các vấn đề kỹ thuật thực tế. Điều này được thực hiện cùng với các yếu tố của e-learning bằng cách cho sinh viên thi công lại các mạch điện tử đã được mô phỏng bằng các phần mềm nêu trên. Cách thực hiện bản mạch (9) vật lý sử dụng dây dẫn, linh kiện và bảng mạch (hình 8).

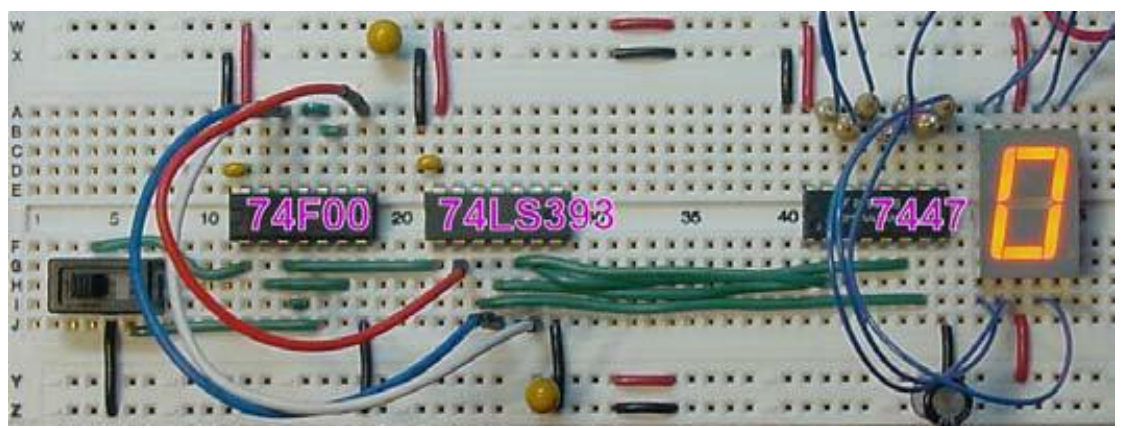

Hình 8. Bảng mạch để xây dụng mạch thực hành

\section{Kết luận}

Một số vấn đề về phương pháp giảng dạy học tập các môn học điện và điện tử đã được thảo luận trong việc áp dụng $\mathrm{CDIO}$ nhằm sửa đổi cách thức học tập và đào tạo các môn học về điện tử tương tự và kỹ thuật số cho chương trình kỹ sư điện - điện tử tại Đại học Công Nghệ Đồng Nai (DNTU). Có ý kiến cho rằng các vấn đề áp dụng các giải pháp elearning cụ thể sẽ đem lại thêm tính tích cực trong hoạt động giảng dạy.

Chúng ta hy vọng rằng mức độ phù hợp của các phương pháp trên, được nhúng trong các tình huống sử dụng có liên quan, có thể được nhấn mạnh hơn trong $\mathrm{CDIO}$, vì nó được coi là một chiến lược phát triển của $\mathrm{CDIO}$ liên quan đến tiêu chuẩn học tập tích cực, tức là CDIO tập trung vào kinh nghiệm thiết kế-xây dựng và thi công các đề tài trên thực tế. 


\section{Tài liệu tham khảo}

[1] Aldeen, M.; Oxbrow, R.: Multimedia enhanced teaching of control systems. IEEE International Conference on Multi Media Engineering Education 1996, 3-5 Jul 1996, pp. 363-371.

[2] Doering, E.R.: Scientific Visualization in the Circuits Curriculum: Enhancing Student Insight ASEE/IEEE Conference Frontiers in Education '95. (http://fie.engrng.pitt.edu/fie95/2c6/2c64/2c64.htm)

[3] Doering, E.R.: CircuitViz: a new method for visualizing the dynamic behavior of electric circuits. IEEE Transactions on Education, 1996, Volume 39(3), pp. 297-303.

[4] Hambley, A. R.: Electrical Engineering. Principles and Applciations. Pearson. Prentice Hall, New Jersey ( $4^{\text {th }}$ Edition), 2008.

[5] Kaminskyi, I.; Mani, N.: A multimedia learning environment for electronic engineering students. IEEE International Conference on Multi Media Engineering Education, 1996, pp. 225 - 230

[6] Lowe, R.: Understanding Information Presented by Complex Animated Diagrams. In: Rouet, J.F., Levonen,J.J., Biardeau, A. (Eds): Multimedia Learning. Cognitive and Instructional Issues. Pergamon/Elsevier \& Earli, Amsterdam 2001.

[7] Meier, R.D.; Wright, C.T., Jr.: The use of simulation based laboratory exercises to improve the undergraduate digital logic design experience. Frontiers in Education Conference FIE '96. 26th Annual Conference., Proceedings of Volume 3, 6-9 Nov. 1996 Page(s):1177 - 1180 vol.3

[8] Price, C.B.: Applications of a Collaborative Open Object Oriented Learning Environment to Electronics, Computing and Mathematics Education. Frontiers in Education FIE '05. Proceedings 35th Annual Conference 19-22 Oct. 2005 pp. F1C-6 - F1C-11

[9] Sutcliffe, A.: On the effective use and reuse of HCI knowledge. ACM Transactions on Computer-Human Interaction (TOCHI), Vol.7 (2) pp. 197-221.

[10] Teh, Y. K.; Mohd-Yasin, F.; Reaz, M. B. I.; Kordesch, A.:A VLSI Design Framework with Freeware CAD Tools. IEEE International Conference on Semiconductor Electronics, 2006, pp. 896-900.

[11] Vahid, F. Digital Design. John Wiley and Sons, New Jersey (2007)

[12] Waks, S.: Mathematics and electronics - the conceptual transfer problem. Physics Education, Vol 23 (4), pp. 234-238. 1988.

[13] 5Spcice software: http://www.5spice.com/

[14] Moodle software: http://www.moodle.org

[15] Circuit Simulator software: http://www.falstad.com

[16] Logisim software: http://sourceforge.net/projects/circuit

[17] Circuit Sandbox software: http://sourceforge.net/projects/circuitsandbox/

[18] Mathematica software: http://www.wolfram.com/ 
